\title{
Electromotive instillation of mitomycin immediately before transurethral resection for patients with primary urothelial non-muscle invasive bladder cancer: a randomised controlled trial
}

Savino M Di Stasi, Marco Valenti, Cristian Verri, Emanuele Liberati, Arcangelo Giurioli, Gioia Leprini, Francesco Masedu, Antonio R Ricci,

Francesco Micali, Giuseppe Vespasiani

\begin{abstract}
Summary
Background The clinical effect of intravesical instillation of chemotherapy immediately after transurethral resection of bladder tumours (TURBT) has recently been questioned, despite its recommendation in guidelines. Our aim was to compare TURBT alone with immediate post-TURBT intravesical passive diffusion (PD) of mitomycin and immediate pre-TURBT intravesical electromotive drug administration (EMDA) of mitomycin in non-muscle invasive bladder cancer.
\end{abstract}

Methods We did a multicentre, randomised, parallel-group study in patients with primary non-muscle invasive bladder cancer in three centres in Italy between Jan 1, 1994, and Dec 31, 2003. Patients were randomly assigned to receive treatment by means of stratified blocked randomisation across six strata. Patients and physicians giving the interventions were aware of assignment, but it was masked from outcome assessors and data analysts. Patients were randomly assigned to receive TURBT alone, immediate post-TURBT instillation of $40 \mathrm{mg}$ PD mitomycin dissolved in $50 \mathrm{~mL}$ sterile water infused over $60 \mathrm{~min}$, or immediate pre-TURBT instillation of $40 \mathrm{mg}$ EMDA mitomycin dissolved in $100 \mathrm{~mL}$ sterile water with intravesical $20 \mathrm{~mA}$ pulsed electric current for $30 \mathrm{~min}$. Our primary endpoints were recurrence rate and disease-free interval. Analyses were done by intention to treat. Follow-up for our trial is complete. This study is registered with ClinicalTrials.gov, number NCT01149174.

Findings 124 patients were randomly assigned to receive TURBT alone, 126 to receive immediate post-TURBT PD mitomycin, and 124 to receive immediate pre-TURBT EMDA mitomycin. 22 patients were excluded from our analyses because they did meet our eligibility criteria after TURBT: 11 had stage pT2 disease and 11 had carcinoma in situ. Median follow-up was 86 months (IQR 57-125). Patients assigned to receive EMDA mitomycin before TURBT had a lower rate of recurrence (44 [38\%] of 117) than those assigned to receive PD mitomycin after TURBT (70 [59\%] of 119) and TURBT alone (74 [64\%] of 116; log-rank $p<0$ - 0001). Patients assigned to receive EMDA mitomycin before TURBT also had a higher disease-free interval (52 months, IQR 32-184) than those assigned to receive PD mitomycin after TURBT (16 months, 12-168) and TURBT alone (12 months, 12-37; log-rank $\mathrm{p}<0 \cdot 0001)$. We recorded persistent bladder symptoms after TURBT in 18 (16\%) of 116 patients in the TURBT-alone group (duration 3-7 days), $37(31 \%)$ of 119 in the PD mitomycin post-TURBT group (duration 20-30 days), and 24 (21\%) of 117 in the EMDA mitomycin pre-TURBT group (duration 7-12 days); haematuria after TURBT in eight (7\%) of 116 patients in the TURBT-alone group, $16(13 \%)$ of 119 in the PD mitomycin post-TURBT group, and $11(9 \%)$ of 117 in the EMDA mitomycin preTURBT group; and bladder perforation after TURBT in five (4\%) of 116 patients in the TURBT-alone group, nine (8\%) of 119 in the PD mitomycin post-TURBT group, and seven (6\%) of 117 in the EMDA mitomycin pre-TURBT group.

Interpretation Intravesical EMDA mitomycin before TURBT is feasible and safe; moreover, it reduces recurrence rates and enhances the disease-free interval compared with intravesical PD mitomycin after TURBT and TURBT alone.

\section{Funding None.}

\section{Introduction}

Bladder cancer is the seventh most common cancer in men. ${ }^{1}$ In 2008 an estimated 386300 cases were diagnosed and 150200 patients died of the disease worldwide. ${ }^{1}$ Of newly diagnosed bladder cancer cases, $75-85 \%$ present as non-muscle invasive disease, including papillary lesions confined to the urothelium (stage Ta) or invading the lamina propria (stage T1), and carcinoma in situ (stage Tis). ${ }^{2}$ Despite adjuvant intravesical therapy after transurethral resection of bladder tumour (TURBT), $31-78 \%$ of patients relapse and $1-45 \%$ progress to muscleinvasive disease within 5 years. ${ }^{3}$ Repeating TURBT and intravesical instillations of immunotherapeutic and chemotherapeutic drugs cause substantial inconvenience and morbidity, making cost per patient from diagnosis to death the highest of all cancers. ${ }^{4}$

One commonly accepted mechanism for tumour recurrence is seeding of circulating tumour cells that are

\author{
Lancet Oncol 2011; 12: 871-79 \\ Published Online \\ August 9, 2011 \\ DOI:10.1016/S1470- \\ 2045(11)70190-5 \\ See Comment page 830 \\ Department of Surgery/ \\ Urology, Tor Vergata \\ University, Rome, Italy \\ (Prof S M Di Stasi MD, C Verri MD, \\ Prof G Vespasiani MD); \\ Operative Unit of Urologic \\ Oncology, Policlinico Casilino, \\ Rome, Italy (Prof S M Di Stasi, \\ ELiberati MD, Prof F Micali MD); \\ Department of Medicine and \\ Public Health, University of \\ L'Aquila, L'Aquila, Italy \\ (Prof M Valenti MD, \\ F Masedu PhD); Operative Unit \\ of Urology, "A Perrino" \\ Hospital, Brindisi, Italy \\ (A Giurioli MD); Medical \\ Biotechnologies, School of \\ Medicine and Surgery and \\ School of Science, Tor Vergata \\ University, Rome, Italy \\ (G Leprini BSC); and Operative \\ Unit of Pathology, "S Pertini" \\ Hospital, Rome, Italy \\ (A R Ricci MD) \\ Correspondence to: \\ Prof Savino M Di Stasi, \\ Department of Surgery/Urology, \\ Tor Vergata University, \\ Via Torrice 4, 00189 Rome, Italy \\ sdistas@tin.it
}


released at resection sites during TURBT. $^{5}$ To kill exfoliated tumour cells that could implant, and thus reduce recurrence, researchers have tested intravesical chemotherapy instillation immediately after TURBT. Meta-analyses by the European Organisation for Research and Treatment of Cancer (EORTC) ${ }^{6}$ and the American Urological Association ${ }^{7}$ reported that intravesical chemotherapy instillation immediately after TURBT reduced recurrence in patients with low-risk non-muscle invasive bladder cancer by $12 \%$ and intermediate risk by $17 \%$ (vs TURBT alone), with negligible side-effects. The European Association of Urology ${ }^{2}$ and, to a lesser extent, the American Urological Association ${ }^{7}$ guidelines consequently recommended that this strategy be included in treatment plans for all patients with non-muscle invasive bladder cancer. However, disagreement persists despite level 1 evidence supporting its use, ${ }^{8-12}$ and it is rarely given by urologists. ${ }^{13}$

In laboratory ${ }^{14}$ and clinical studies, ${ }^{15}$ intravesical electromotive drug administration (EMDA) increased mitomycin bladder uptake, improving clinical efficacy in high-risk non-muscle invasive bladder cancer. Intravesical EMDA mitomycin is not recommended immediately after TURBT because catheter electrode rigidity might cause additional mechanical trauma and urothelial injury, leading to bladder spasm and leakage of drug solution. Furthermore, haematuria and bladder perforation are contraindications to intravesical EMDA mitomycin. Our aim was to compare the effects of intravesical EMDA mitomycin immediately before TURBT, intravesical passive diffusion (PD) mitomycin immediately after TURBT, and TURBT alone in patients with primary urothelial non-muscle invasive bladder cancer.

\section{Methods}

\section{Participants}

Between Jan 1, 1994, and Dec 31, 2003, we enrolled patients into our multicentre, randomised, parallel-group study at three centres in Italy: the Department of Surgery/ Urology of Tor Vergata University of Rome, which coordinated and did the study; the Urology Oncology Unit, Policlinico Casilino, Rome, Italy and the Urology Unit, “A Perrino" Hospital, Brindisi, Italy, from which the participants were recruited. All patients underwent abdominal ultrasound, urinary cytology, and cystoscopy with cold-cup biopsy of the bladder tumours. We graded all tumour biopsy samples with the 1973 WHO classifications. ${ }^{16}$ We assessed risk categories for recurrence and progression in accordance with Guidelines of the European Association of Urology for non-muscle invasive bladder cancer. ${ }^{2}$

Eligible participants had histologically proven primary pTa and pT1 urothelial carcinoma of the bladder. Our inclusion criteria were age 18 years or older, adequate bone-marrow reserve (ie, white-blood-cell count $\geq 4000 \times 10^{6}$ cells per L; platelet count $\geq 120 \times 10^{9} / \mathrm{L}$ ), normal renal function (ie, serum creatinine $\leq 123.76 \mu \mathrm{mol} / \mathrm{L}$ ), normal liver function (ie, serum glutamic-oxaloacetic aminotransferase $\leq 42 \mathrm{U} / \mathrm{L}$, serum glutamic-pyruvic aminotransferase $\leq 48 \mathrm{U} / \mathrm{L}$, and total bilirubin $\leq 22 \mu \mathrm{mol} / \mathrm{L}$ ), and Eastern Cooperative Oncology Group performance status between 0 and 2. ${ }^{17}$ Our exclusion criteria were non-urothelial carcinomas of the bladder; previous bladder cancer; previous intravesical treatment with chemotherapeutic and immunotherapeutic drugs; known allergy to mitomycin; previous or concomitant urinary-tract carcinoma in situ, urothelial carcinoma of the upper urinary tract and urethra, or both; bladder capacity less than $200 \mathrm{~mL}$; untreated urinary-tract infection; severe systemic infection (ie, sepsis); treatment with immunosuppressive drugs; urethral strictures that would prevent endoscopic procedures and catheterisation; previous radiotherapy to the pelvis; other concurrent chemotherapy, radiotherapy, and treatment with biological response modifiers; other malignant diseases within 5 years of trial registration (except for adequately treated basal-cell or squamous-cell skin cancer, in situ cervical cancer); pregnancy; and any factors that would preclude study participation. All enrolled patients signed an informed consent form, approved by the institutional review boards, providing details of treatments. The institutional review boards of each participating centre approved the study design.

\section{Randomisation and masking}

We randomly assigned patients to our study groups within 7 days of cystoscopy, cold-cup bladder tumour biopsy, and urinary cytology. We used tumour number and histology for stratification. We randomly assigned patients to one of our three treatment groups by means of stratified-blocked randomisation across six (two by three) strata derived from two prognostic criteria: unifocal versus multifocal tumours, and grade 1 versus grade 2 versus grade 3 urothelial carcinoma. This method ensured prognostic parity among our three treatment groups.

In each stratum, given two possible block lengths of three and six respectively, with a random number generator giving numbers uniformly distributed between 0 and 1 , we selected a block length of three for random numbers lower than 0.5 or a block length of six otherwise. We then did a random permutation procedure by choosing a random number to associate with each of the letters ("ABC" or "AABBCC", where A, B, and C respectively are the three treatment groups) of a block and then assigning, in order, the letter ranked by the corresponding random number. The procedure was repeated for each of the six strata.

We concealed assignment by varying the blocking number. Variation of the block number prevented the clinician from guessing what the next treatment will be; therefore, varying the block sizes at random makes it difficult (although not impossible) to break the treatment code. The assignment sequence was generated by the 
statistical unit of the study, which subsequently did the masked data analysis. Participants were enrolled by the clinical operative units. Patients were assigned each time to the trial group by the epidemiological assessor of the study. Patients and the physicians giving the interventions were aware of assignment; however, assignment was masked from the outcome assessors and data analysts.

\section{Procedure}

Patients received interventions about 2 weeks after randomisation. For TURBT alone, patients underwent TURBT of all bladder tumours visible on endoscopy, ensuring that muscle was included in resected samples, as specified by European Association of Urology guidelines. ${ }^{2}$ For intravesical PD mitomycin immediately after TURBT, patients received $40 \mathrm{mg}$ mitomycin dissolved in $50 \mathrm{~mL}$ sterile water within $6 \mathrm{~h}$ of TURBT. After bladder draining, the mitomycin solution was infused intravesically through a Foley catheter, retained in the bladder for $60 \mathrm{~min}$ with catheter clamping, and then drained. After bladder draining the patient received continuous intravesical irrigation with saline for $8 \mathrm{~h}$ (daysurgery admission) to $18 \mathrm{~h}$ (elective admission). When persistent macroscopic haematuria was present, bladder perforation was suspected or noted during TURBT, or both, post-TURBT intravesical PD mitomycin was avoided, but patients were followed up and included in our analysis.

For immediately pre-TURBT intravesical EMDA mitomycin, about $30 \mathrm{~min}$ before spinal or general anaesthesia TURBT patients received $40 \mathrm{mg}$ mitomycin dissolved in $100 \mathrm{~mL}$ sterile water, ${ }^{15,18}$ infused intravesically through the Foley catheter by gravity and retained in the bladder for $30 \mathrm{~min}$, while $20 \mathrm{~mA}$ pulsed electric current for $30 \mathrm{~min}$ was given externally. The mitomycin solution was drained and TURBT was done, subsequently continuous intravesical irrigation with saline for 8-18 h, to prevent clot formation or retention and related complications. As previously described, ${ }^{19}$ intravesical EMDA is given by a battery-powered generator delivering a controlled electric current that passes between the active intravesical electrode (integrated into a specific transurethral catheter) and dispersive ground electrodes (on skin of the lower abdomen). Operators set active electrode polarity and current intensity on the generator. No patient underwent cystogram assessment after TURBT. Patients in the mitomycin groups were placed on fluid restriction without urine alkalinisation to prevent changes in acid-base balance during and after anaesthesia.

Tumours were staged in accordance with the 1997 TNM classification of the Union for International Cancer Control. ${ }^{20}$ About a month after TURBT, patients without muscle in resected samples, positive or suspect cytology, carcinoma in situ, stage T1, or grade 3 tumours underwent restaging transurethral resection, random cold-cup bladder and prostatic urethra biopsies (ie, sampling of seemingly healthy urothelium and suspicious areas), and upper-urinary-tract imaging. All tumour and bladder biopsy samples were reviewed by a reference pathologist (AR) for histology, grade, and stage.

No adjuvant intravesical therapy was given to patients with low-risk non-muscle invasive bladder cancer. All patients with intermediate-risk and high-risk non-muscle invasive bladder cancer in our three groups were scheduled to receive adjuvant long-term intravesical therapy starting about 3 weeks after TURBT procedures. Patients with intermediate-risk disease received an initial intravesical treatment of $40 \mathrm{mg}$ PD mitomycin dissolved in $50 \mathrm{~mL}$ sterile water and retained in the bladder for $60 \mathrm{~min}$, once a week for 6 weeks. Patients with high-risk disease received an initial intravesical treatment of $81 \mathrm{mg}$ BCG Connaught substrain (ImmuCyst, Alfa Wassermann SpA, Bologna, Italy) dissolved in $50 \mathrm{~mL}$ bacteriostatic-free solution of $0.9 \%$ sodium chloride and retained in the bladder for 120 min once a week for 6 weeks. Patients assigned to receive $\mathrm{BCG}$ and mitomycin were placed on fluid restriction and $2 \mathrm{~g}$ ingested sodium bicarbonate the night before treatment, the morning of treatment, and $2 \mathrm{~h}$ before treatment with mitomycin. Patients who were disease-free 3 months after induction treatment were scheduled to receive monthly intravesical instillation for 10 months, with the same dose and methods of infusion as initial assigned treatment. Thus, a total of 16 instillations were given over 12 months. In case of recurrence, TURBT was done and maintenance mitomycin was continued in accordance with the initial schedule in patients with intermediate-risk disease, whereas a second 6-week course of BCG was given to patients with high-risk disease. We suspended patients on adjuvant intravesical mitomycin or BCG from the trial on a second recurrence, development of carcinoma in situ, development of carcinoma in the upper urinary tract or prostatic urethra, progression to muscle-invasive disease (ie, stage pT2 or more advanced), or the development of metastases. Further treatment was left to the discretion of the local investigator.

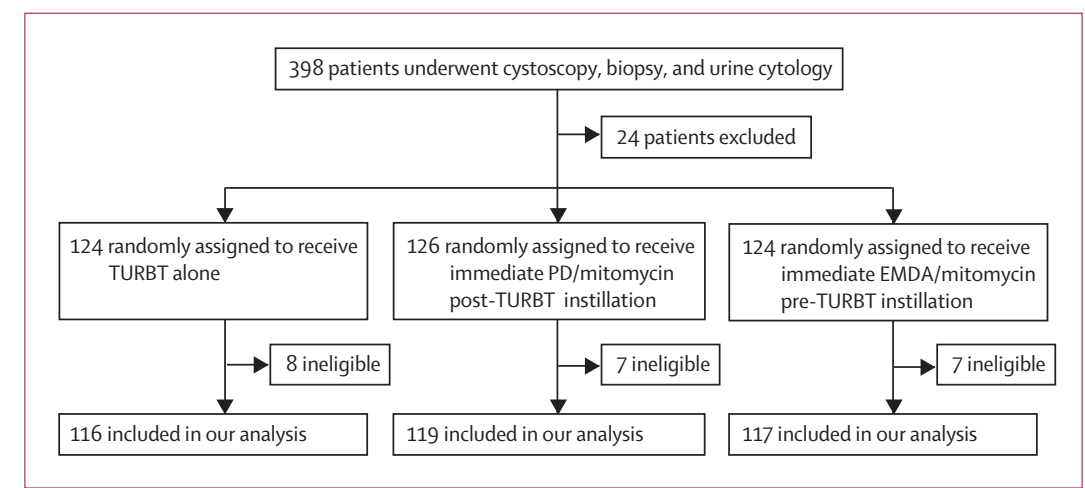

Figure 1: Trial profile

The ineligible patients were excluded because they did not fit our criteria after TURBT: 11 had stage pT2 disease (four TURBT alone, three PD mitomycin post-TURBT, four EMDA mitomycin pre-TURBT), and 11 had carcinoma in situ (four TURBT alone, four PD mitomycin post-TURBT, three EMDA mitomycin pre-TURBT). TURBT=transurethral resection of bladder tumours. $\mathrm{PD}=$ passive diffusion. $\mathrm{EMDA}=$ electromotive drug administration. 
Our primary endpoints were recurrence rate and disease-free interval for patients who were disease-free after treatment-ie, time from randomisation to the first cystoscopy noting recurrence. Our secondary endpoints were time to progression (ie, time from randomisation until onset of muscle-invasive disease [stage T2 or higher] as recorded by pathological assessment of TURBT or biopsy samples), overall survival (ie, time from randomisation until death from any cause), and disease-

\begin{tabular}{|c|c|c|c|}
\hline & $\begin{array}{l}\text { TURBT alone } \\
(n=116)\end{array}$ & $\begin{array}{l}\text { PD mitomycin after } \\
\text { TURBT }(n=119)\end{array}$ & $\begin{array}{l}\text { EMDA mitomycin } \\
\text { before TURBT }(n=117)\end{array}$ \\
\hline Number of women (\%) & $24(21 \%)$ & $27(23 \%)$ & $25(21 \%)$ \\
\hline Number of men (\%) & $92(79 \%)$ & $92(77 \%)$ & $92(79 \%)$ \\
\hline Median age, years (IQR) & $66 \cdot 5(60 \cdot 0-73 \cdot 0)$ & $67 \cdot 0(61 \cdot 0-72 \cdot 0)$ & $67 \cdot 0(63 \cdot 0-74 \cdot 0)$ \\
\hline Median follow-up, months (IQR) & $92 \cdot 0(61 \cdot 0-126 \cdot 0)$ & $82 \cdot 0(50 \cdot 0-125 \cdot 0)$ & $85 \cdot 0(57 \cdot 0-126 \cdot 0)$ \\
\hline \multicolumn{4}{|c|}{ Number with disease characteristics (\%) } \\
\hline $\mathrm{pTa} / \mathrm{G} 1$ unifocal & $9(8 \%)$ & $10(8 \%)$ & $11(9 \%)$ \\
\hline pTa/G1 multifocal & $12(10 \%)$ & $13(11 \%)$ & $11(9 \%)$ \\
\hline $\mathrm{pTa} / \mathrm{G} 2$ unifocal & $18(16 \%)$ & $17(14 \%)$ & $16(14 \%)$ \\
\hline pTa/G2 multifocal & $24(21 \%)$ & $24(20 \%)$ & $25(21 \%)$ \\
\hline pT1/G2 unifocal & $4(3 \%)$ & $3(3 \%)$ & $3(3 \%)$ \\
\hline pT1/G2 multifocal & $17(15 \%)$ & $20(17 \%)$ & $18(15 \%)$ \\
\hline pT1/G3 unifocal & $5(4 \%)$ & $5(4 \%)$ & $6(5 \%)$ \\
\hline pT1/G3 multifocal & $27(23 \%)$ & $27(23 \%)$ & $27(23 \%)$ \\
\hline \multicolumn{4}{|c|}{ Number in risk categories in accordance with EAU guidelines (\%) } \\
\hline Low (all unifocal) & $9(8 \%)$ & $10(8 \%)$ & $11(9 \%)$ \\
\hline Intermediate & $75(65 \%)$ & $77(65 \%)$ & $73(62 \%)$ \\
\hline Unifocal & $22(19 \%)$ & $20(17 \%)$ & $19(16 \%)$ \\
\hline Multifocal & $53(46 \%)$ & $57(48 \%)$ & $54(46 \%)$ \\
\hline High & $32(28 \%)$ & $32(27 \%)$ & $33(28 \%)$ \\
\hline Unifocal & $5(4 \%)$ & $5(4 \%)$ & $6(5 \%)$ \\
\hline Multifocal & $27(23 \%)$ & $27(23 \%)$ & $27(23 \%)$ \\
\hline \multicolumn{4}{|c|}{$\begin{array}{l}\text { TURBT=transurethral resection of bladder tumours. PD=passive diffusion. EMDA=electromotive drug administration. } \\
\text { EAU=European Association of Urology. }\end{array}$} \\
\hline
\end{tabular}

specific survival (ie, time from randomisation until death from bladder cancer). We censored patients without recurrence or progression at the last cystoscopy and dropouts on the last known day of survival.

Response to treatment was assessed with abdominal ultrasonography, cystoscopy, and urinary cytology. Patients who were disease-free 3 months after treatment were assessed every 3 months for the first 3 years, twice during the third year, and yearly thereafter. At cystoscopy any tumour or abnormal looking urothelium was resected and tissue sent to the reference pathologist to confirm recurrence. All patients were assessed for safety. Symptoms and side-effects, classified as local, systemic, or allergic, were recorded during and after each perioperative (EMDA mitomycin pre-TURBT and PD mitomycin post-TURBT) and adjuvant (PD mitomycin in patients with intermediate-risk disease and BCG in patients with high-risk disease) intravesical treatment. Local side-effects were defined as culture-proven bacterial cystitis, drug-induced (ie, chemical) cystitis, visible haematuria, dysuria, prostatitis, epididymitis, obstruction of the ureter, and contracted bladder; systemic sideeffects as fever of greater than $38^{\circ} \mathrm{C}$, general malaise and fatigue, liver toxicity, BCG-induced lung infection, and BCG sepsis; and allergic side-effects as skin rash, arthralgia, and arthritis. The severity of symptoms and side-effects was classified by the attending physician as those that needed no delay, delay, or indefinite postponement of intravesical treatment.

\section{Statistical analysis}

Given previous reports ${ }^{21}$ and our own experience, we assumed the 5-year recurrence-free probability to be about $60 \%$ in patients with non-muscle invasive bladder cancer treated with intravesical therapy; we assumed a $20 \%$ increase in time to recurrence was clinically relevant.

The sample size had to be about 297 to detect-with power $1-\beta=0.80$ and type 1 error $\alpha=0.05$-if the three

\begin{tabular}{|c|c|c|c|c|c|c|}
\hline & $\begin{array}{l}\text { TURBT alone } \\
(n=116)\end{array}$ & $\begin{array}{l}\text { PD mitomycin } \\
\text { after TURBT } \\
(n=119)\end{array}$ & $\begin{array}{l}\text { EMDA mitomycin } \\
\text { before TURBT } \\
(n=117)\end{array}$ & $\begin{array}{l}\text { PD mitomycin after } \\
\text { TURBT vs TURBT alone, } \\
\mathrm{HR}^{*}(95 \% \mathrm{Cl})\end{array}$ & $\begin{array}{l}\text { EMDA mitomycin } \\
\text { before TURBT vs TURBT } \\
\text { alone, } \mathrm{HR}^{*}(95 \% \mathrm{CI})\end{array}$ & $\begin{array}{l}\text { Log-rank } \\
\text { p value }\end{array}$ \\
\hline Overall $(n=352)$ & $74 / 116(64 \%)$ & $70 / 119(59 \%)$ & 44/117 (38\%) & $0.84(0.61-1.17)$ & $0.40(0.28-0.59)$ & $<0.0001$ \\
\hline Unifocal ( $n=107)$ & $18 / 36(50 \%)$ & $15 / 35(43 \%)$ & $10 / 36(28 \%)$ & $0.78(0.39-1.55)$ & $0.43(0.20-0.94)$ & 0.08 \\
\hline Multifocal $(n=245)$ & $56 / 80(70 \%)$ & $55 / 84(66 \%)$ & $34 / 81(42 \%)$ & $0.84(0.58-1.22)$ & $0.38(0.25-0.58)$ & $<0.0001$ \\
\hline Low risk $(n=30)$ & $0 / 9(0 \%)$ & $0 / 10(0 \%)$ & $1 / 11(9 \%)$ & .. & .. & 0.39 \\
\hline Intermediate risk $(\mathrm{n}=225)$ & $47 / 75(63 \%)$ & $46 / 77(60 \%)$ & 26/73 (36\%) & $0.87(0.58-1.30)$ & $0.40(0.25-0.65)$ & 0.0004 \\
\hline Unifocal $(n=61)$ & $14 / 22(64 \%)$ & $12 / 20(60 \%)$ & $6 / 19(32 \%)$ & $0.83(0.38-1.79)$ & $0.35(0.13-0.92)$ & 0.08 \\
\hline Multifocal (n=164) & $33 / 53(62 \%)$ & $34 / 57(60 \%)$ & $20 / 54(37 \%)$ & $0.85(0.53-1.38)$ & $0.41(0.24-0.72)$ & 0.004 \\
\hline High risk ( $n=97$ ) & $27 / 32(84 \%)$ & 24/32 (75\%) & $17 / 33(52 \%)$ & $0.81(0.47-1.41)$ & $0.30(0.16-0.55)$ & 0.0002 \\
\hline Unifocal $(n=16)$ & $4 / 5(80 \%)$ & $3 / 5(60 \%)$ & $3 / 6(50 \%)$ & $0.69(0.15-3.12)$ & $0.32(0.07-1.49)$ & $0 \cdot 32$ \\
\hline Multifocal $(n=81)$ & $23 / 27(85 \%)$ & $21 / 27(78 \%)$ & $14 / 27(52 \%)$ & $0.84(0.47-1.53)$ & $0.30(0.15-0.58)$ & 0.0005 \\
\hline
\end{tabular}


treatments differed by the difference between the arcsin square-root transformations of both expected and known recurrence-free proportions. Allowance for 15\% potential withdrawal increased the sample to about 342 -ie, 114 patients per treatment group. More than 120 recurrences were needed to ensure at least $80 \%$ power to detect a hazard ratio (HR) between any two treatments that might exceed $2 \cdot 0$, with a log-rank test at $\alpha=0 \cdot 05$.

We did all analyses by intention to treat. We estimated times to first recurrence and progression, overall survival, and disease-specific survival with a generalised k-sample log-rank test. All tests were two-sided; we deemed $\mathrm{p}$ values below 0.05 significant. We calculated HRs with $95 \%$ CI by proportional-hazards regression. We did our statistical analyses with SAS/STAT software (version 9.2). This study is registered with ClinicalTrials. gov, number NCT01149174.

\section{Role of the funding source}

There was no funding source for this study. The corresponding author had full access to all the data in the study and had final responsibility for the decision to submit for publication.

\section{Results}

We screened 398 patients at our three centres. Before randomisation we excluded 24 patients because of concomitant transitional cell carcinoma of the upper urinary tract (five patients) and prostatic urethra (seven), histology other than pure urothelial carcinoma (eight), and refusal of consent (four). Thus, 374 patients were randomly assigned to receive treatment (figure 1). Restaging transurethral resection and random cold-cup bladder and prostatic urethra biopsies were necessary in 112 patients: 97 with high-risk disease and 15 with multifocal intermediate-risk disease. On restaging TURBT, histological findings showed that 12 patients with residual stage pTa or pT1 tumour were eligible, whereas 11 with concomitant pTis and 11 with muscleinvasive disease (stage pT2) were ineligible. In total, we included 352 patients in our analysis. Pertinent characteristics in our three treatment groups were similar (table 1). 225 patients with intermediate-risk disease received adjuvant long-term intravesical mitomycin (75 in the TURBT-alone group [compliance 73 of 75], 77 in the PD mitomycin post-TURBT group [72 of 77], and 73 in the EMDA mitomycin pre-TURBT group [74 of 75]) and 97 with high-risk disease received BCG (32 in the TURBTalone group [compliance 30 of 32], 32 in the PD mitomycin post-TURBT group [31 of 32], and 33 in the EMDA mitomycin pre-TURBT group [30 of 33]).

After a median follow-up of 86 months (IQR 57-125), $42(36 \%)$ of 116 patient treated with TURBT alone, 49 (41\%) of 119 treated with PD mitomycin after TURBT, and $73(62 \%)$ of 117 treated with EMDA mitomycin before TURBT were disease-free $(\mathrm{p}<0 \cdot 0001)$; significantly fewer patients treated with EMDA mitomycin before TURBT had recurrences than did those who were treated with either TURBT alone or with PD mitomycin after TURBT (table 2).

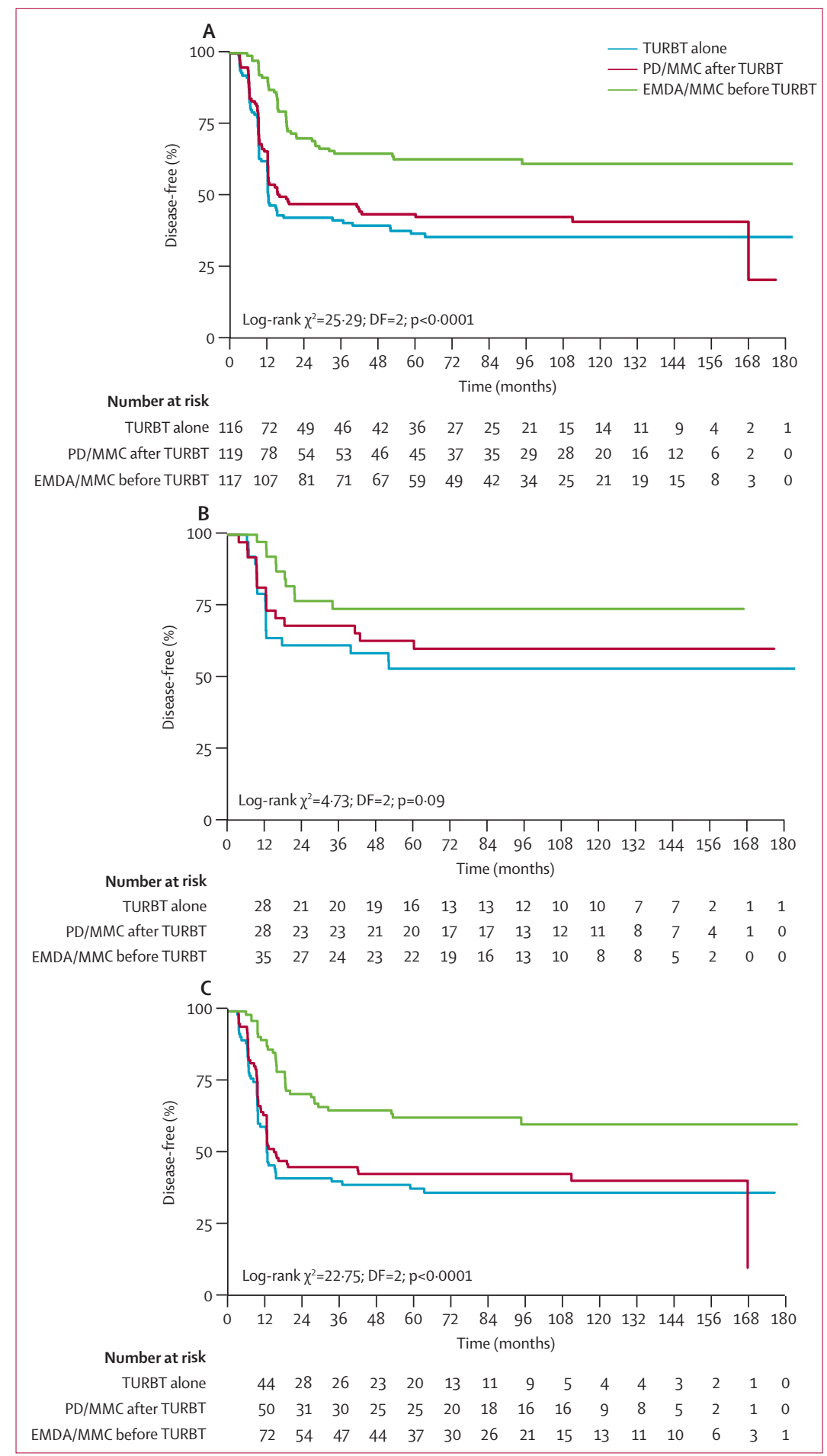

Figure 2: Disease-free interval overall (A), in unifocal disease (B), and in multifocal disease (C)

TURBT=transurethral resection of bladder tumours. $P D=$ passive diffusion. $M M C=$ mitomycin. $E M D A=$ electromotive drug administration. 


\begin{tabular}{|clll|}
\hline & $\begin{array}{l}\text { TURBT alone } \\
(\mathrm{n}=\mathbf{1 1 6})\end{array}$ & $\begin{array}{l}\text { PD mitomycin after } \\
\text { TURBT }(\mathrm{n}=\mathbf{1 1 9})\end{array}$ & $\begin{array}{l}\text { EMDA mitomycin before } \\
\text { TURBT }(\mathrm{n}=117)\end{array}$ \\
\hline Overall $(\mathrm{n}=352)$ & $12(12-37)$ & $16(12-168)$ & $52(32-184)^{*}$ \\
Unifocal $(\mathrm{n}=107)$ & $52(12-\mathrm{NA})$ & NA $(18-\mathrm{NA})$ & NA \\
Multifocal $(\mathrm{n}=245)$ & $13(9-15)$ & $12(11-19)$ & NA $(52-\mathrm{NA})$ \\
Low risk $(\mathrm{n}=30)$ & $\mathrm{NA}$ & NA & NA \\
Intermediate risk $(\mathrm{n}=225)$ & $15(12-58)$ & $19(12-167)$ & NA \\
Unifocal $(\mathrm{n}=61)$ & $29(12-\mathrm{NA})$ & $41(12-\mathrm{NA})$ & NA (21-NA) \\
Multifocal $(\mathrm{n}=164)$ & $12(9-63)$ & $18(12-168)$ & NA (94-NA) \\
High risk $(\mathrm{n}=97)$ & $10(9-12)$ & $11(6-12)$ & $27(15-\mathrm{NA})$ \\
Unifocal $(\mathrm{n}=16)$ & $12(6-\mathrm{NA})$ & $9(3-\mathrm{NA})$ & NA (15-NA) \\
Multifocal $(\mathrm{n}=81)$ & $9(9-12)$ & $12(6-12)$ & $28(15-\mathrm{NA})$ \\
\end{tabular}

Data are months (IQR). TURBT=transurethral resection of bladder tumours. PD=passive diffusion.

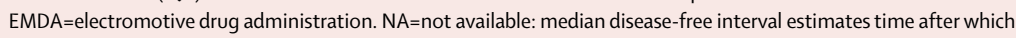
$50 \%$ of study group are expected to survive; for diseases with favourable prognosis, survival probability might not reach 0.5 during study period, preventing estimate of median time or upper $95 \% \mathrm{Cl}$. *With respect to overall data in the EMDA mitomycin pre-TURBT treatment group, median time was not estimated, but calculated from the dataset.

Table 3: Median disease-free interval by treatment group

There were fewer recurrences in patients with multifocal intermediate-risk and high-risk disease who received an intravesical EMDA mitomycin instillation before TURBT. Pairwise comparisons showed a significant difference between EMDA mitomycin before TURBT and TURBT alone (HR 0 40, 95\% CI 0 28-0 - 59), and no significant difference between TURBT alone and PD mitomycin after TURBT in the overall population $(0 \cdot 84,0 \cdot 61-1 \cdot 17$; table 2$)$. Similar findings were noted in intermediate-risk $(0 \cdot 41,0 \cdot 24-0 \cdot 72)$ and high-risk $(0 \cdot 30$, $0 \cdot 15-0.58)$ patients with multifocal tumours (table 2 ). No differences between groups were noted for patients with low-risk disease because of the low event rate in this subgroup (table 2).

The disease-free interval was longer after EMDA mitomycin before TURBT (52 months, IQR 32-184) than after PD mitomycin after TURBT (16 months, 12-168) or TURBT alone (12 months, 12-37; log-rank $\mathrm{p}<0 \cdot 0001$; figure 2 and table 3 ).

$24(21 \%)$ of 116 patients treated with TURBT alone, 23 (19\%) of 119 treated with PD mitomycin after TURBT, and $19(16 \%)$ of 117 treated with EMDA mitomycin before TURBT progressed to muscle-invasive disease (log-rank $\mathrm{p}=0 \cdot 55)$. There was no disease progression in patients with low-risk disease, but 28 (12\%) of 225 with intermediate-risk disease and 37 (38\%) of 97 with highrisk disease progressed $(\log$-rank $\mathrm{p}=0 \cdot 66)$.

We did not identify significant differences in overall survival (50 [43\%] of 116 patients treated with TURBT alone, 58 [49\%] of 119 treated with PD mitomycin after TURBT, and 54 [46\%] of 117 treated with EMDA mitomycin before TURBT had died; log-rank $\mathrm{p}=0 \cdot 66$ ), and diseasespecific survival (15 [13\%] of 116 treated with TURBT alone, 15 [13\%] of 119 treated with PD mitomycin after TURBT, and 11 [9\%] of 117 treated with EMDA mitomycin before TURBT had died of disease-specific causes; logrank $\mathrm{p}=0.62$ ).
For TURBT alone, we detected overt bladder perforation in five (4\%) of 116 patients and gross haematuria in eight $(7 \%)$ of 116 . Irritative bladder symptoms, lasting 3-7 days after resection, were reported in $18(16 \%)$ of 116 . For PD mitomycin after TURBT, intravesical instillation was stopped after 10-15 $\min$ in 28 (24\%) of 119 patients because of pain, bladder spasm, and leakage of drug solution. After TURBT in this group, overt bladder perforation was detected in nine (8\%) of 119 patients and gross haematuria in $16(13 \%)$ of 119 . Irritative bladder symptoms, lasting 20-30 days after resection, were reported in 37 (31\%) of 119. For EMDA mitomycin before TURBT, 116 (99\%) of 117 patients completed treatment. Intravesical instillation was not given to one patient because of urethral stricture; nine $(8 \%)$ of 117 had transient mild (pink) haematuria after instillation. After TURBT in this group, we detected overt bladder perforation in seven $(6 \%)$ of 117 patients and gross haematuria in $11(9 \%)$ of 117 . Irritative bladder symptoms, lasting 7-12 days after resection, were reported in 24 (21\%) of 117 . In both perioperative mitomycin groups there were no allergic reactions or haematological toxic effects.

In intermediate-risk patients who underwent adjuvant intravesical mitomycin, we recorded local side-effects and symptoms, including chemical cystitis, visible haematuria, and urinary frequency in 16 (21\%) of 75 patients in the TURBT-alone group, in $19(25 \%)$ of 77 in the PD mitomycin post-TURBT group, and in $19(26 \%)$ of 73 in the EMDA mitomycin pre-TURBT group ( $\mathrm{p}=0 \cdot 47)$. All other local side-effects were noted in less than $1 \%$ of the treated patients. Treatment was stopped because of chemical cystitis side-effects in two $(3 \%)$ of 75 , three $(4 \%)$ of 77 , and one $(1 \%)$ of 73 patients of these groups, respectively.

In high-risk patients who underwent adjuvant intravesical BCG, we recorded local side-effects and symptoms in $18(56 \%)$ of 32 patients in the TURBTalone group, in 17 (53\%) of 32 in the PD mitomycin post-TURBT group, and in 18 (55\%) of 33 in the EMDA mitomycin pre-TURBT group $(\mathrm{p}=0 \cdot 87)$. We noted other local side-effects, including granulomatous prostatitis, epididymitis, and ureteral obstruction, in less than 3\% of the treated patients. Six (6\%) of 97 patients did not complete the first 6 weekly treatments because of severe toxic effects, including two $(6 \%)$ of 32 in the TURBTalone group because of prostatitis and persistent visible haematuria, one $(3 \%)$ of 32 in the PD mitomycin postTURBT group because of recurrent fever of $39^{\circ} \mathrm{C}$ or higher, drug-induced cystitis, and persistent and systemic side-effects, and three (9\%) of 33 in the EMDA mitomycin group because of drug-induced cystitis, persistent general malaise, and arthralgia. We did not record any haematological toxic effects, no patients had a contracted bladder, and there were no life-threatening adverse effects or treatment-related deaths. 


\section{Discussion}

Our findings show that intravesical instillation of EMDA mitomycin given immediately before TURBT in patients with primary non-muscle invasive bladder cancer provided significant long-term benefits: the recurrence rate was lower and median disease-free interval longer with EMDA mitomycin before TURBT than after intravesical PD mitomycin after TURBT or TURBT alone. We noted improvements in all stratification groups in intermediate-risk and high-risk non-muscle invasive bladder cancer, and significantly in patients with multifocal disease. We noted no differences between groups for patients with low-risk disease, primarily because only one of 30 patients had disease recurrence.

EMDA mitomycin before TURBT gives suboptimal benefit in unifocal intermediate-risk and high-risk disease, for which no significant difference was shown between recurrence rates in the PD mitomycin postTURBT group and the TURBT-alone group. Recurrence rates and disease-free intervals did not differ significantly after PD mitomycin after TURBT and TURBT alone.

Although we did not do cost analyses during the course of the study, we must highlight the potential economic gains of our treatment protocol. In our 5-year follow-up, EMDA mitomycin before TURBT provided a $21 \%$ absolute reduction in recurrence compared with $\mathrm{PD}$ mitomycin after TURBT. This change corresponds to a number needed to treat of 4.8 people per recurrence averted, which is lower than that calculated in previous meta-analyses of intravesical instillation immediately after TURBT. ${ }^{22}$ Since progression to muscle-invasive disease, death, or both, did not differ significantly between our three groups, our data suggest that perioperative intravesical instillation of chemotherapy plus intravesical standard regimens (eg, BCG or chemotherapy) are insufficient for patients at risk of disease progression. Even though attempts to develop drugs for BCG-refractory non-muscle invasive bladder cancer continue, none so far have shown high rates of long-term success. ${ }^{23}$ Intravesical sequential BCG and EMDA mitomycin compared with BCG alone in highrisk non-muscle invasive bladder cancer was associated with significantly fewer disease recurrences, progressions, and deaths. ${ }^{24}$ If confirmed in controlled multicentre clinical trials, the improved outcomes, particularly compared with failed intravesical treatment in high-risk disease, would justify the cost of intravesical EMDA therapy. Costs will inevitably need to be scrutinised, even though the initial expense of the present generator (often supplied free by the manufacturer) and the running costs of disposables (electrode catheter and indifferent electrodes) seems slight-ie, about $€ 180$ on average throughout Europe.

As reported elsewhere,$^{15}$ intravesical EMDA increases bladder uptake of mitomycin, improving efficacy in highrisk non-muscle invasive bladder cancer, compared with
Panel: Research in context

\section{Systematic review}

One commonly accepted mechanism for tumour recurrence in bladder cancer is re-implantation of floating tumour cells that are released during transurethral resection. Meta-analyses by the European Organisation for Research and Treatment of Cancer ${ }^{6}$ and the American Urological Association ${ }^{7}$ reported that a single dose of intravesical chemotherapy a few hours after transurethral resection reduced recurrence in patients with low-risk and intermediate-risk non-muscle invasive bladder cancer, with negligible side-effects. Despite level 1 evidence supporting its use, however, disagreement persists, ${ }^{8-12}$ and it is rarely given by urologists. Moreover, it has suboptimum efficacy in intermediate-risk patients and no benefit in high-risk patients, and there are other difficulties: the optimum schedule and duration of treatment are not yet standardised. Sometimes patients do not tolerate the procedure for the full course of treatment, and spasms, leakage, and haematuria cause premature evacuation of the drug, which probably decreases its potential benefits; if there is a bladder perforation during transurethral resection, it can result in severe complications because of extravesical extravasation of the drugs.

\section{Interpretation}

So far, all clinical trials designed to address the efficacy of intravesical passive diffusion (PD) mitomycin after transurethral resection have shown sufficient evidence of an advantage versus transurethral resection alone. We show that intravesical electromotive drug administration (EMDA) mitomycin before transurethral resection can be introduced in clinical practice as an alternative, safe treatment for patients with urothelial nonmuscle invasive bladder cancer. Intravesical EMDA mitomycin before transurethral resection ensures a higher and deeper bladder target tissue concentration than intravesical PD mitomycin after transurethral resection. Intravesical EMDA mitomycin is an easily manageable procedure that is safe and well tolerated by patients. We show that intravesical EMDA mitomycin reduces recurrence and enhances the disease-free interval in intermediate-risk and high-risk non-muscle invasive bladder cancer; it has also proven to be efficacious in patients with multifocal disease, unlike either transurethral resection alone or PD mitomycin after transurethral resection. Clearly, intravesical EMDA mitomycin before transurethral resection or intravesical PD mitomycin after transurethra resection might be redundant in low-risk patients: a well done transurethral resection alone should ensure no recurrence and avoid the need for adjuvant treatments in general. We recommend further research to confirm intravesical EMDA mitomycin before transurethral resection as a standard treatment in non-muscle invasive bladder cancer. In our opinion, intravesical EMDA mitomycin is the most effective and rational way to give intravesical mitomycin and our findings show that EMDA mitomycin before transurethral resection is the safest perioperative treatment for non-muscle invasive bladder cancer.

passive mitomycin transport. Our findings suggest that high mitomycin concentrations in bladder tissue destroyed or inhibited implantation of viable cancer cells that could have been released during TURBT and thus reduced the recurrence rate of non-muscle invasive bladder cancer, with no long-term loss of treatment efficacy. An alternative but less probable hypothesis is that intravesical EMDA mitomycin before TURBT is able to treat residual (unrecognised and hence unresected or incompletely resected) tumour and the deeper tissue, which is exposed after TURBT. Intravesical peripoerative chemotherapy cannot compensate for an incomplete TURBT and this emphasises the need for careful endoscopic examination of the bladder and attention to detail during endoscopic tumour resection. 
At present, the European Association of Urology has included intravesical instillation of PD chemotherapy immediately after TURBT as standard of care for all patients with non-muscle invasive bladder cancer, whereas the American Urological Association recommends it as a treatment option (panel). ${ }^{7}$ Underlying these recommendations is a meta-analysis of seven randomised trials with 1476 patients, ${ }^{5}$ showing recurrences dropped from $48.4 \%$ after TURBT alone to $36.7 \%$ after intravesical instillation of chemotherapy was added immediately after TURBT. Evidence was high-level for primary, unifocal, low-grade disease, and borderline for multifocal disease.

Several papers have recently suggested that chemotherapy instillation immediately after TURBT should be abandoned in non-muscle invasive bladder cancer because it prevented only small tumour recurrences; ${ }^{9}$ was of limited benefit in patients with multifocal, rapidly recurrent, high-grade disease, and did not seem to increase the efficacy of subsequent protracted intravesical regimens; ${ }^{10-12}$ was efficacious mainly during the first 2 years of follow-up; ${ }^{25}$ and had no effect on disease progression. ${ }^{6,11}$

In a randomised study, ${ }^{12}$ intravesical instillation of gemcitabine immediately after TURBT was no better than placebo in terms of recurrence-free survival. Continuous bladder irrigation with saline solution and improved TURBT techniques were postulated to underlie the low recurrence rate. ${ }^{13}$ Our findings reject the continuous bladder irrigation hypothesis, because all patients received continuous bladder irrigation with saline solution after TURBT for 8-18 h. The role of early intravesical instillation of chemotherapy after TURBT with fluorescence cystoscopy has not been established. However, even if disease detection and resection completeness are improved, ${ }^{2}$ the risk of tumour-cell seeding remains.

To prevent tumour-cell implantation, the use of distilled water has been recommended. ${ }^{26}$ Cytotoxic effects of distilled water are mainly caused by osmotic cytolysis. Invitro studies provide evidence of distilled water impairing bladder cancer reimplantation ${ }^{26}$ and have similar effects to $\mathrm{BCG}^{27}$ and mitomycin ${ }^{28}$ in bladder-cancer cells. However, when these effects are transferred to an in-vivo model there are several issues: tumour cell compounds might not be safely accessible to osmotic damage, and osmotic effects on healthy bladder mucosa are unclear. In the EORTC randomised trial,,$^{29}$ the effect of an early single instillation of epirubicin $80 \mathrm{mg}$ in $50 \mathrm{~mL}$ saline versus $50 \mathrm{~mL}$ sterile water was assessed in 399 patients at low risk for recurrence. The interval to initial recurrence was significantly in favour of the epirubicin group and the recurrence rate per year reduced by nearly $50 \%$ after a mean follow-up of 2 years. A recent in-vitro study ${ }^{30}$ showed that a solution of $20 \%$ polyethylene glycol 400 in water is a promising irrigant with antiadhesive and cytotoxic properties, which could be used to prevent tumour cell reimplantation during TURBT.
Our study, of course, has limitations. A major concern about the design of our study is not having a group in receipt of intravesical PD mitomycin before TURBT. However, laboratory ${ }^{15}$ and clinica ${ }^{16}$ studies showed that mitomycin concentrations in the bladder-wall tissues were significantly increased by applying pulsed electric current (EMDA). Higher drug concentrations might have a clinical effect since intravesical EMDA mitomycin was associated with a lower recurrence rate than PD mitomycin. Further, in our study, cystoscopy, biopsy, and urinary cytology before randomisation constituted an additional step (with related costs and harms) with respect to standard protocols for immediate post-TURBT chemotherapy. However, it ensured preliminary patient stratification and homogeneous groups for assessment. Additionally, the endoscopic impression is often inaccurate, ${ }^{31}$ and erroneous tumour status assessment inevitably leads to opting for treatments with no potential value. As to the broader use of the techniques we propose, a potential weakness is that, despite evidence, urologists might be reluctant to practise additional manoeuvres for mitomycin administration. We hope that this position will change rapidly as evidence on cost-effectiveness emerges.

We showed that intravesical EMDA mitomycin before TURBT is feasible and safe. In other studies, the safety profile of intravesical chemotherapy instillation immediately after TURBT was usually not presented or associated with insignificant morbidity; ${ }^{6,11}$ rare but severe toxic effects were reported after deep resection or overt bladder perforation. ${ }^{32,33}$ Our findings show that intravesical PD mitomycin immediately after TURBT was associated with persistent lower urinary-tract symptoms exceeding those recorded after TURBT alone and those reported in randomised trials and metaanalyses. ${ }^{6,11}$ Some degree of drug extravasion in patients undergoing complete, correct TURBT remains unrecognised because cystography is not routine. ${ }^{34}$ This might have contributed to the high rate of persistent lower urinary-tract symptoms in our study.

For intravesical non-muscle invasive bladder cancer therapy, understanding the pharmacology of chemotherapeutic drugs and strategies to maximise drug delivery might aid in the design of rational clinical trials to provide patients with the best treatment for long-term cancer control. New frameworks for treatment of nonmuscle invasive bladder cancer-eg, sequential intravesical BCG and EMDA mitomycin, as well as intravesical EMDA mitomycin immediately before TURBT-have provided promising preliminary results with higher remission rates and longer remission times, and they are a priority to minimise the costs of disease management.

In conclusion, when managing non-muscle invasive bladder cancer, the role of EMDA needs to be assessed in depth and the advantages of intravesical EMDA mitomycin should not be ignored. We hope that future developments will enable intravesical EMDA mitomycin to be added in standardised treatment protocols. 


\section{Contributors}

SMDS was the principal investigator and provided primary conception, design, logistics, and treatment protocols. MV coordinated the randomisation process and data analysis. $\mathrm{CV}, \mathrm{EL}, \mathrm{AG}, \mathrm{GV}$, and $\mathrm{FM}$ recruited and treated patients. GL organised data collection and checked the quality of data. FMa managed statistical analyses. AR reviewed all biopsy samples of tumour and bladder. All authors participated in writing and reviewing the paper, and accept full responsibility for its overall content.

Conflicts of interest

We declare that we have no conflicts of interest.

\section{Acknowledgments}

This paper is dedicated to the memory of Robert (Bob) L Stephen who was an inspiring teacher, scientist, colleague, friend, and mentor. Our study was supported by state grants to "Tor Vergata" University of Rome, Italy. We thank Maurizio Brausi for his help in data interpretation and Cino Rossi for his collaboration and technical assistance.

\section{References}

1 Jemal A, Bray F, Center MM, Ferlay J, Ward E, Forman D. Global cancer statistics. Ca Cancer J Clin 2011; 61: 69-90.

2 Babjuk M, Oosterlinck W, Sylvester R, et al. EAU guidelines on non-muscle invasive urothelial carcinoma of the bladder, the 2011 update. Eur Urol 2011; 59: 997-1008.

3 Sylvester RJ, van der Meijden AP, Oosterlinck W, et al. Predicting recurrence and progression in individual patients with stage Ta T1 bladder cancer using EORTC risk tables: a combined analysis of 2596 patients from seven EORTC trials. Eur Urol 2006; 49: 466-75.

4 Botteman MF, Pashos CL, Redaelli A, Laskin B, Hauser R. The health economics of bladder cancer: a comprehensive review of the published literature. Pharmacoeconomic 2003; 21: 1315-30.

5 Weldon TE, Soloway MS. Susceptibility of urothelium to neoplastic cellular implantation. Urology 1975; 5: 824-27.

6 Sylvester RJ, Oosterlinck W, van der Mejiden APM. A single immediate postoperative instillation of chemotherapy decreases the risk of recurrence in patients with stage Ta T1 bladder cancer: a meta-analysis of published results of randomized clinical trials. J Urol 2004; 171: 2186-90.

7 Hall MC, Chang SS, Dalbagni G, et al. Guideline for the management of nonmuscle invasive bladder cancer (stages Ta, T1, and Tis): 2007 update. J Urol 2007; 178: 2314-30.

8 Berrum-Svennung I, Gransfors T, Jahnson S, Boman H, Holmang S. A single instillation of epirubicin after transurethral resection of bladder tumours prevent only small recurrences. J Urol 2008; 179: 101-06.

9 Gudjónsson S, Adell L, Merdasa F, et al. Should all patients with non-muscle-invasive bladder cancer receive early intravesical chemotherapy after transurethral resection? The results of a prospective randomised multicentre study. Eur Urol 2009; 55: 773-80.

10 Dobruch J, Herr H. Should all patients receive single chemotherapeutic agent instillation after bladder tumour resection? BJU Int 2009; 104: 170-74.

11 Cai T, Nesi G, Tinacci G, et al. Can early single dose instillation of epirubicin improve bacillus Calmette-Guerin efficacy in patients with nonmuscle invasive high risk bladder cancer? Results from a prospective, randomized, double-blind controlled study. J Urol 2008; 180: 110-15.

12 Böhle A, Leyh H, Frei C, et al. Single postoperative instillation of gemcitabine in patients with non-muscle-invasive transitional cell carcinoma of the bladder: a randomised, double-blind, placebo-controlled phase III multicentre study. Eur Urol 2009. 56: 495-503.

13 O'Donnel MA. When medical evidence alone is simply not enough. Cancer 2009; 115: 2602-604.

14 Di Stasi SM, Giannantoni A, Massoud R, et al. Electromotive versus passive diffusion of mitomycin $\mathrm{C}$ into human bladder wall: concentration-depth profiles studies. Cancer Res 1999; 59: 4912-18.

15 Di Stasi SM, Giannantoni A, Stephen RL, et al. Intravesical electromotive mitomycin $\mathrm{C}$ vs passive transport mitomycin $\mathrm{C}$ for high risk superficial bladder cancer: a prospective randomized study. J Urol 2003; 170: 777-82.
16 Mostofi FK, Sobin LH, Torloniu H. Histological typing of urinary bladder tumours: International Classification of Tumours, No 10. Geneva: World Health Organization, 1973.

17 Oken MM, Creech RH, Tormey DC, et al. Toxicity and response criteria of the Eastern Cooperative Oncology Group. Am J Clin Oncol 1928; 5: 649-55.

18 Gontero P, Cattel L, Paone TC, et al. Pharmacokinetic study to optimize the intravesical administration of gemcitabine. BJU Int 2010; 106: 1652-56.

19 Di Stasi SM, Riedl C. Updates in intravesical electromotive drug administration of mitomycin-C for non-muscle invasive bladder cancer. World J Urol 2009; 27: 325-30.

20 Sobin LH, Wittekind CH. UICC (International Union Against Cancer): TNM classification of malignant tumours, 5th edn New York, NY: Wiley Liss, 1997: 107-90.

21 Lamm DL, Riggs DR, Traynelis CL, Nseyo UO. Apparent failure of current intravesical chemotherapy prophylaxis to influence the long-term course of superficial transitional cell carcinoma of the bladder. J Urol 1995; 153: 1444-50.

22 Feifer A, Xuanqian X, Brophy JM, Segal R, Kassouf W. Contemporary cost analysis of single instillation of mytomicin after transurethral resection of bladder tumour in a universal health care system. Urology 2010; 76: 652-57.

23 Morgan TM, Keegan KA, Clark PE. Bladder cancer. Curr Opin Oncol 2011; 23: 275-82.

24 Di Stasi SM, Giannantoni A, Capelli G, et al. Sequential BCG and electromotive mitomycin versus BCG alone for high risk superficial bladder cancer: a randomised controlled trial. Lancet Oncol 2006, 7: 43-51.

25 Solsona E, Iborra I, Ricos JV, Monros JL, Casanova J, Dumont R. Effectiveness of single immediate mitomycin $\mathrm{C}$ instillation in patients with low risk superficial bladder cancer: short and long-term follow-up. J Urol 1999; 161: 1120-23.

26 Levin DR, Moskovitz B. Distilled water versus chemotherapeutic agents for transitional bladder carcinoma. Eur Urol 1986; 12: 418-21.

27 Bolkier M, Moskovitz B, Ginesin Y, Levin DR. Effect of distilled water and bacillus Calmette-Guerin on exfoliated bladder tumor cells. Eur Urol 1991; 19: 319-21.

28 Fechner G, Pocha K, Schmidt D, Müller SC. Reducing recurrence and costs in superficial bladder cancer: preclinical evaluation of osmotic cytolysis by distilled water $v s$ mitomycin. Int J Clin Pract 2006; 60: 1178-80.

29 Oosterlinck W, Kurth KH, Schröder F, Bultinck J, Hammond B, Sylvester R. A prospective European Organization for Research and Treatment of Cancer Genitourinary Group randomized trial comparing transurethral resection followed by single intravesical instillation of epirubicin or water in single stage Ta, T1 papillary carcinoma of the bladder. J Urol 1993; 149: 749-52.

30 Sangeda RZ, Vandepitte J, Huygens A, Van Cleynenbreugel B, Van Poppel H, de Witte PA. Prevention of tumor cell reimplantation during transurethral resection: the in-vitro antiadhesive and cytotoxic properties of an irrigant containing polyethylene glycol 400. Anticancer Drugs 2010; 21: 645-50.

31 Cina SJ, Epstein JI, Endrizzi JM, Harmon WJ, Schoenberg MP. Consideration of cystoscopic impression with histologic diagnosis of biopsy specimens of the bladder. Hum Pathol 2001; 32: 630-37.

32 Shapiro O, Jones K, Wang C, Landas S, Haas GP. Risk of postoperative intravesical mitomycin $\mathrm{C}$ instillation following transurethral bladder tumor resection. Can J Urol 2006; 13: 3317-320.

33 Tyritzis SI, Stravodimos KG, Mihalakis A, Costantinides CA. Complications associated with primary and secondary perforation of the bladder following immediate instillations of epirubicin after transurethral resection of superficial urothelial tumours. Int Urol Nephrol 2009; 41: 865-68.

34 Balbay MD, Çimentepe E, Ünsal A, Bayrak Ö, Koç A, Akbulut Z The actual incidence of bladder perforation following transhrethral bladder surgery. J Urol 2005; 174: 2260-63. 